\title{
Standounkt
}

\section{Die Lebensdauer von Produkten ist planbar}

\author{
Die Entwicklung zuverlässiger und somit langlebiger \\ Produkte sollte für Unternehmen eine Selbstverständlich- \\ keit sein. Zum einen hängt die Reputation des Unter- \\ nehmens stark davon ab, zum andern ist es eine Frage \\ der Wirtschaftsethik. Von Janis Winzer
}

$\mathbf{S}$ pätestens seit dem Dokumentarfilm „Kaufen für die Müllhalde“ und dem Buch "Made to Break" (2006) ist eine gesellschaftliche Diskussion über Obsoleszenz (Kurzlebigkeit von Produkten) entstanden. Als Folge der gesellschaftlichen Auseinandersetzung mit Obsoleszenz ist sehr schnell der Murks? Nein Danke! e.V. gegründet worden, erste Sachbücher wurden geschrieben und die Wissenschaft begann mit der seit 1976 ruhenden Forschung.

Schon die Zeitschrift Advertising \& Selling druckte 1929 erste Auszüge von J.George Frederick aus dem Buch seiner Frau "Selling Mrs. Consumer" hinsichtlich Lösungsstrategien gegen die Konsumstagnation. Die Vorschläge liberaler Kreise sahen vor, der Bevölkerung einfach mehr Geld in die Hand zu geben. J. G. Frederick sah diese Maßnahme als „unzulänglichen Notbehelf“ an und sprach sich dagegen aus. Als einen wirksameren Hebel sah er die mit dem Kunstwort "progressive Obsoleszenz" bezeichnete Idee an. Zusammengefasst besagt sein Vorschlag, man müsse den Menschen beibringen, mehr Waren auf Grundlage einer Leistungs-, Wirtschaftlichkeits-, Mode- oder Geschmacksobsoleszenz zu kaufen. Erstmals als "geplante Obsoleszenz" publiziert, wurde das Phänomen durch Bernard London in seinem 1933 veröffentlichten Werk The New Prosperity. Londons Wirtschaftstheorie ist rein ökonomisch betrachtet, sie basiert jedoch auf der Annahme von grenzenlosem Wirtschaftswachstum und unendlich vorkommenden natürli- chen Ressourcen. Nach Londons Theorie sollten alle Produkte mit einer Art "Verfallsdatum“ versehen werden. Nach Ablauf dieses Datums sollten die Produkte als amtlich "tot" gelten und von staatlicher Stelle „zerstört“ werden. Das Behalten der Produkte über ihr Verfallsdatum hinaus sollte unter Strafe gestellt werden.

\section{Haben und sein}

Neben den wirtschaftlichen und technischen Aspekten haben aber auch das intrinsische Streben des Menschen nach Mehr und soziale Praktiken einen großen Einfluss auf Obsoleszenz. So geht bspw. Paul Gilbert davon aus, dass die westlichen Gesellschaften inzwischen sehr stark darauf fixiert sind, die Emotionen des Antriebssystems zu verstärken. Dieses Streben und und der Drang, etwas zu besitzen, ist für viele wie eine Sucht, was zum Teil dazu führt, dass wir unser Nervensystem ständig überstimulieren und unter dauerhafter Dopaminausschüttung leiden. Es gibt demzufolge Menschen, die sich nur dann gut fühlen, wenn sie ständig etwas erreichen oder ein Verlangen durch Kauf befriedigen.

Neben den psychologischen Theorien gehen andere davon aus, dass das Handeln von Menschen weder durch die Motive und Wünsche des Individuums bestimmt werden noch allein durch gesellschaftliche Strukturen. Die Entscheidung für ein neues Produkt wird eingebettet in bestimmte Regeln des Handelns, in das, was als „normal“, was als „regelkonform“ erachtet wird, getroffen. Treiber für Obsoleszenz bestehen demzufolge auch abseits von intrinsischer Motivation und unternehmerischen Produktionslogiken, sie bestehen auch in sozialen Praktiken - vielfach in Form von Routinen.

\section{Kurze Produktlebensdauern}

Die Möglichkeiten des Staates, gegen Obsoleszenz vorzugehen, sind nach Jahrzehnten der auf Ludwig Erhards Theorem zurückgehenden Konsensualpolitik auf nationaler Ebene und nach den Regeln des Komitologieverfahrens auf europäischer Ebene zwar stark begrenzt, aber nicht gänzlich verloren gegangen. Insbesondere die Ökodesign-Richtlinie kann in den kommenden Jahren Maßnahmen gegen Obsoleszenz in Kraft setzen. Erste Ansätze zeigt die EU-Verordnung für Staubsauger (Nr. 666/2013), in der ab 09/2017 eine Mindestlebensdauer von 500 Stunden für den Elektromotor gefordert ist. Politisch leiten sich diese gesetzgeberischen Maßnahmen bisher aus der Energie- und Ressourceneffizienzpolitik ab. Die steigende Intention des Gesetzgebers hinsichtlich Ressourceneffizienzpolitik wurzelt in der integrierten Produktpolitik. Die Forschung hat die Umweltprobleme, ausgelöst von Produkten, inzwischen sehr deutlich aufgezeigt.

Neben dem Verlust der sogenannten grauen Energie für Rohstoffgewinnung und Herstellung, können Produkte bei Weitem noch nicht in geschlossenen Kreisläufen geführt werden. Da die Ressourceneffizienz mit steigenden Produktlebensdauern zunimmt, ist die Politik neben Herstellern und Konsument/ innen auch weiter gefordert, dem Phänomen Obsoleszenz wirksam entgegenzuwirken.

AUTOREN + KONTAKT

Dr. Janis Winzer ist wissenschaftlicher Mitarbeiter am Fraunhofer IZM und Co-Leiter der BMBF-Nachwuchsgruppe Obsoleszenz.

Fraunhofer IZM Gustav-Meyer-Allee 25, 13355 Berlin. Tel.: +49 30464037984

E-Mail: janis.winzer@izm.fraunhofer.de and redistributing the material in any medium or format, provided the original work is properly cited, it is not used for commercial purposes and it is not remixed, transformed or built upon. The access to the digital version of this article is reserved to subscribers of ÖkologischesWirtschaften until two years after the date of publication; after two years it is available to all readers. 